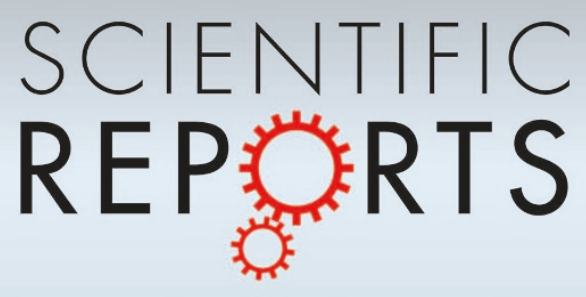

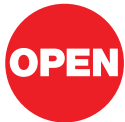

SUBJECT AREAS: ANIMAL PHYSIOLOGY

PHYSIOLOGY

MOLECULAR EVOLUTION

ENVIRONMENTAL SCIENCES

Received

19 February 2013

Accepted

16 April 2013

Published

14 August 2013

Correspondence and requests for materials should be addressed to

N.S. (ns@med.

kanazawa-u.ac.jp, nsns@staff.kanazawa-u.

ac.jp)

\section{Upregulation of aquaporin expression in the salivary glands of heat-acclimated}

\section{rats}

\author{
Naotoshi Sugimoto ${ }^{1,2}$, Kentaro Matsuzaki $^{2}$, Hiroaki Ishibashi ${ }^{3}$, Masao Tanaka $^{4}$, Toshioki Sawaki ${ }^{4}$, \\ Yoshimasa Fujita ${ }^{4}$, Takafumi Kawanami ${ }^{4}$, Yasufumi Masaki ${ }^{4}$, Toshiro Okazaki ${ }^{4}$, Joji Sekine ${ }^{3}$, \\ Shoichi Koizumi ${ }^{5}$, Akihiro Yachie ${ }^{6}$, Hisanori Umehara ${ }^{4} \&$ Osamu Shido
}

'Department of Physiology, Graduate School of Medical Science, Kanazawa University, ${ }^{2}$ Department of Environmental Physiology, School of Medicine, Shimane University, ${ }^{3}$ Department of Oral and Maxillofacial Surgery, Shimane University Faculty of Medicine, ${ }^{4}$ Department of Hematology and Immunology, Kanazawa Medical University, ${ }^{5}$ United Graduate School of Child Development, Graduate School of Medical Science, Kanazawa University, ${ }^{6}$ Department of Pediatrics, Graduate School of Medical Science, Kanazawa University.

It is known that aquaporin (AQP) 5 expression in the apical membrane of acinar cells in salivary glands is important for the secretion of saliva in rodents and humans. Although heat acclimation enhances saliva secretion in rodents, the molecular mechanism of how heat induces saliva secretion has not been determined. Here, we found that heat acclimation enhanced the expression of AQP5 and AQP1 in rat submandibular glands concomitant with the promotion of the HIF-1 $\alpha$ pathway, leading to VEGF induction and CD31-positive angiogenesis. The apical membrane distribution of AQP5 in serous acinar cells enhanced after heat acclimation, while AQP1 expression was restricted to the endothelial cells in the submandibular glands. A network of AQPs may be involved in heat-acclimated regulation in saliva secretion. Because AQPs probably plays a crucial role in saliva secretion in humans, these findings may lead to a novel strategy for treating saliva hyposecretion.

T o suppress the increases in body temperature under heat, humans, monkeys, and horses enhance heat dissipation by sweat evaporation as well as cutaneous vasodilation ${ }^{1-3}$. However, many mammals do not sweat for thermoregulation, and can enhance their heat dissipation by panting or salivation ${ }^{4,5}$. In rodents such as rats and mice, saliva can substitute for sweat for application to their body trunk during rises in body or environmental temperature ${ }^{5,6}$. Although the salivary secretion of rats and mice is regulated by the autonomic nervous system ${ }^{7,8}$, there are salivary secretory mechanisms that might not be explained by autonomic nerve activity alone such as, the water permeability of the salivary acinar cells ${ }^{9,10}$.

Saliva is an important oral fluid critical for the preservation and maintenance of oral health ${ }^{11}$. Although saliva is composed of a variety of electrolytes, immunoglobulins, and enzymes, it is a very dilute fluid composed of more than $99 \%$ water $^{12}$. The acinar cells have high membrane-water permeability and are a likely site of aquaporin (AQP) expression ${ }^{13}$. AQPs are transmembrane proteins that constitute a family of water channel molecules. They are generally responsible for rapid osmotic water movement across the plasma membrane in the secretory and absorptive cells of many species of plants, bacteria, yeast, and mammals ${ }^{14,15}$. They are expressed in various epithelial tissues where they function as channels allowing the permeability of water and small solutes. Subsets of epithelia in which AQP water channels are expressed have a 10- to 100-times greater capacity for water permeation than those in which these proteins are not expressed ${ }^{16}$. To date, at least 13 members of this channel family have been identified in mammals, which are classified into 4 subgroups. AQP0, AQP1, AQP2, AQP4, AQP5, and AQP6 facilitate the transport of water only, while AQP3, AQP7, AQP9, and AQP10 allow the transport of glycerol and urea as well as water. AQP8 might also facilitate the transport of urea in addition to water ${ }^{16,17}$. AQP11 and 12 belong to a new subfamily termed as superaquaporins ${ }^{18}$.

CD31 is a cell surface marker for endothelial cells of microvasculature, hypoxic inducible factor-1 (HIF-1) pathway including VEGF is a major contributor to angiogenesis ${ }^{19,20}$. Interestingly, AQP1 is known to be restricted to the endothelial cells of microvasculature in salivary glands, whereas AQP5 has previously detected in the acinar cells of salivary glands in adult humans, mice, and rats ${ }^{10,21,22}$. AQP5-deficient mice exhibit defective saliva 
secretion ${ }^{10}$ because of the decreased permeability of salivary acinar cell membranes to water ${ }^{9}$. In addition, AQP5 has been shown to be misdirected to the basal membranes of acinus in the salivary glands of a patient with Sjögern's syndrome ${ }^{23}$, a chronic autoimmune disease characterized by lymphocytic infiltration of the exocrine glands resulting in saliva hyposecretion and dry eyes. As per these evidences, it is very probable that AQP5 plays a crucial role in saliva secretion in both humans and rodents.

Organisms have undergone natural selection to deal with thermal fluctuations in ambient environment to obtain well-developed defense and adaptation machineries. When animals encounter heat stress, they experience active responses such as induction of signal pathways and reprogrammed gene expression to retune their internal milieu. The most well-characterized heat shock response is the induction of a highly conserved set of polypeptides termed as heat shock proteins (HSPs). HSPs confer cytoprotection via molecules called chaperones that assist in the correct folding of proteins or the degradation of abnormal proteins or, alternatively, via their facilitatory interactions with cytoprotective molecular signaling pathways $^{24-26}$. Heat acclimation has several effects on HSPs, including accelerating the transcriptional response and increasing the cellular reserves of inducible HSPs species ${ }^{27}$.

Rats in ambient temperatures $\left(\mathrm{T}_{\mathrm{a}}\right)$ higher than $32^{\circ} \mathrm{C}$ exhibit the activation of salivary secretion for evaporative heat loss via the autonomic nervous system ${ }^{28}$. Furthermore, prolonged exposure of rats to moderate heat results in heat acclimation, including a significant enlargement of the salivary gland and remarkable increase in saliva volume ${ }^{28,29}$. Changes in glandular responsiveness during heat acclimation may originate peripherally, thereby changing the enlargement of the salivary glands ${ }^{30}$. However, the mechanisms of enhancement of heat dissipation by saliva after heat acclimation may not be fully explained by the enhancement of autonomic nerve a

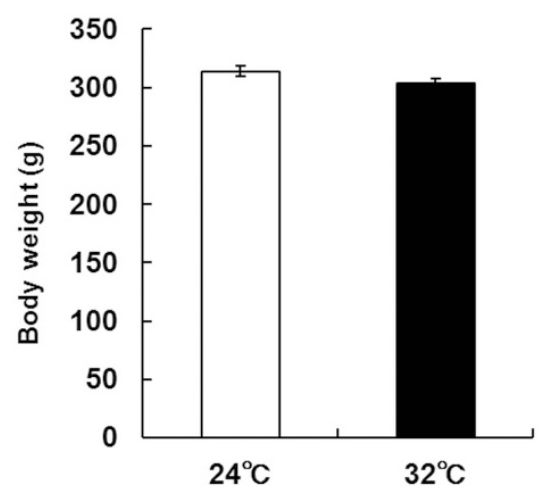

C

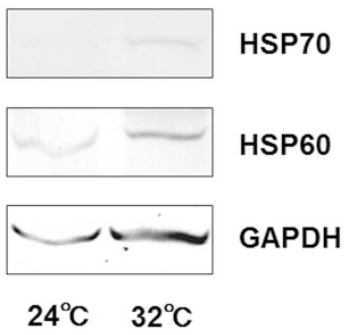

b submandibular glands

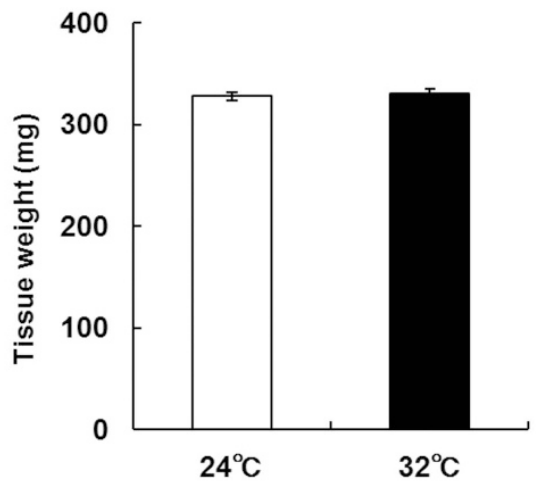

d
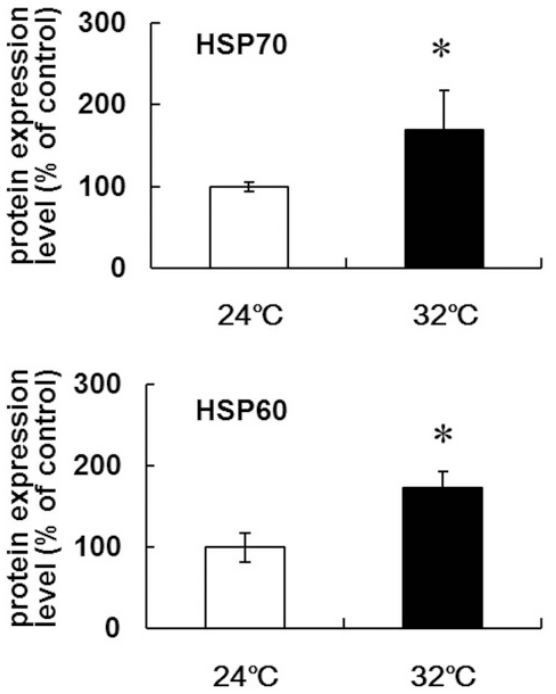

Figure 1 HSP60 and HSP70 expression in rat submandibular glands is upregulated after 5 days of continuous exposure to a $\mathrm{T}_{\mathrm{a}}$ of $32^{\circ} \mathrm{C}$. (a) Body weights of rats kept at a $\mathrm{T}_{\mathrm{a}}$ of $24^{\circ} \mathrm{C}$ and $32^{\circ} \mathrm{C}$ for 5 days. Values are the means \pm SEM. (b) Tissue weights of submandibular glands in rats kept at a $\mathrm{T}_{\mathrm{a}}$ of $24^{\circ} \mathrm{C}$ or $32^{\circ} \mathrm{C}$ for 5 days. Values are the means \pm SEM. (c) Western blotting of HSP 60 and HSP70 in the submandibular glands of rats kept at a $\mathrm{T}_{\mathrm{a}}$ of $24^{\circ} \mathrm{C}$ or $32^{\circ} \mathrm{C}$ for 5 days. (d) Relative levels of protein expression in the submandibular glands of rats kept at a $\mathrm{T}_{\mathrm{a}}$ of $24^{\circ} \mathrm{C}$ or $32^{\circ} \mathrm{C}$ for 5 days. Values are the means \pm SEM. ${ }^{*} \mathrm{P}<0.05$. 
activity and the morphological changes in the salivary glands. The molecular mechanisms underlying increased saliva secretion after heat acclimation have not been determined. Although AQPs are important water channels for salivary secretion, the expression profiles of AQP family members in the salivary glands after heat acclimation have not been determined.

Based on the previous findings, we hypothesized that heat acclimation might induce saliva secretion through a mechanism involving the regulation of AQP expression. The present study aimed to investigate the changes in the AQP expression of the submandibular gland in heat-acclimated rats, which revealed a major secretory molecular mechanism of heat acclimation in vivo.

\section{Results}

Body weights, submandibular gland weights, and heat shock proteins. Body weights and submandibular gland tissue weights did not differ between the 5-day continuous heat exposure in the study rats and control rats (Fig. 1A, B). In vivo experiments have shown that HSP levels increase in several organs in mice exposed to 5 days of heat ${ }^{31}$ and in peripheral blood mononuclear cells in humans ${ }^{32}$. Similar to the results of previous studies, this study showed that 5 days of exposure to $32^{\circ} \mathrm{C}$ increased the levels of HSP60 and HSP70 in the submandibular glands of rats (Fig. 1C, D).

Expression of aquaporins in submandibular glands. To test whether AQPs are involved in the regulation of saliva secretion in rats exposed to continuous heat, we first examined the expression of several AQP mRNAs in submandibular glands using RT-PCR. The mRNA expression of AQP1, AQP5, and AQP8 was detectable in the control rats and in the rats exposed to continuous heat, but that of other AQPs was below the limit of detection (Fig. 2A). The mRNA expression levels of AQP1 and AQP5, but not of AQP8, significantly increased at 5 days after continuous heat exposure in rats (Fig. 2B, C). To confirm the results of RT-PCR, we next examined the expression of AQP1 and AQP5 in submandibular glands by western blotting. Anti-AQP1 and anti-AQP5 antibodies are available for detecting rat AQP1 and AQP5, respectively, whereas anti-AQP8 antibody is unavailable. As expected, AQP1 and AQP5 protein levels increased at 5 days after continuous heat exposure (Fig. 3). These results suggest that continuous heat exposure may regulate the expression of AQP1 and AQP5 for adaptation to heat in the submandibular glands of rats.

Cellular distribution of AQP1 in the submandibular glands of rats. To better understand the function of AQP1 and AQP5 in the submandibular glands, it is important to study the cellular distribution of the corresponding AQP proteins.

In a previous study, Akamatsu et al. suggested that AQP1 is localized in blood vessels, including many capillaries, and is not detectable in the acinar or duct cells of rat submandibular glands ${ }^{21}$. In this study, AQP1 was detected and localized at the capillaries that are distinguishable morphologically in the submandibular glands (Fig. 4A). AQP1-positive cells in the submandibular glands increased

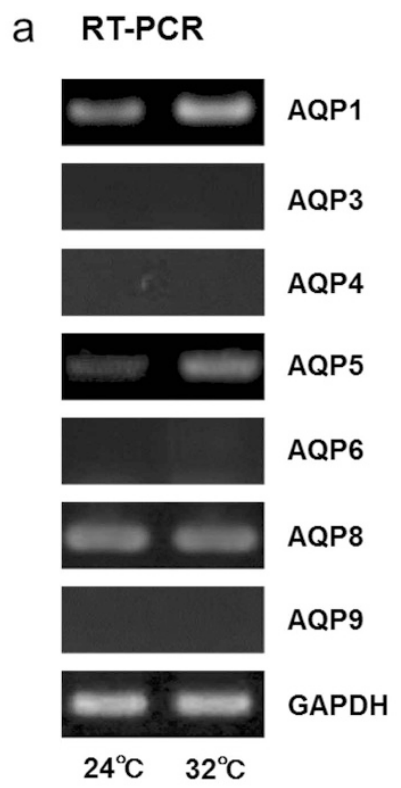

b
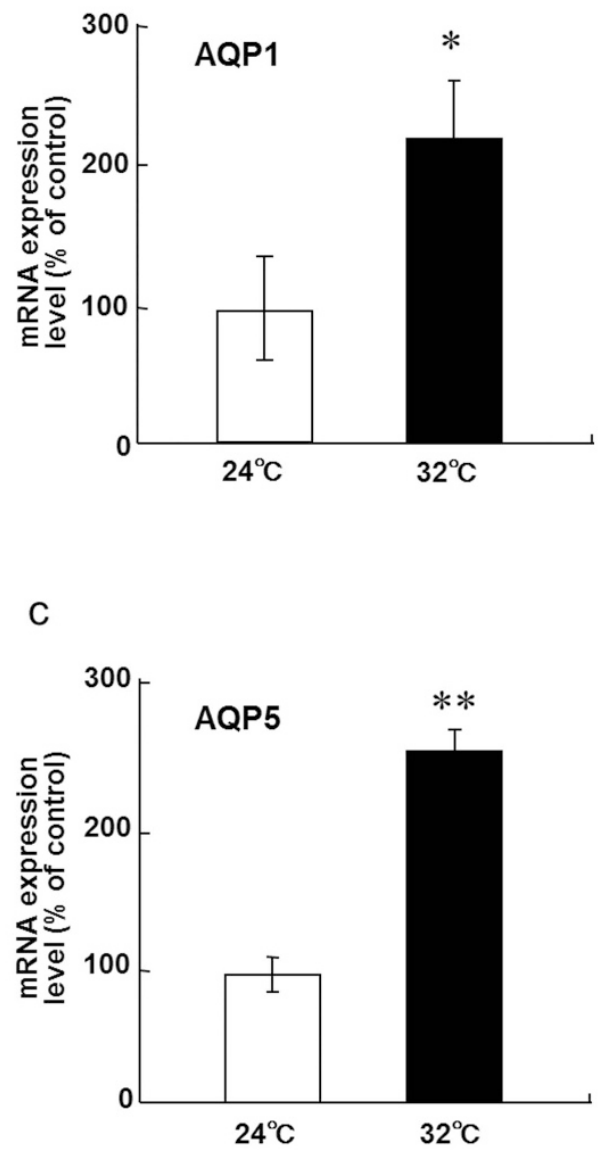

Figure $2 \mid$ mRNA levels of AQP1 and AQP5 in rat submandibular glands are upregulated after 5 days of continuous exposure to a $\mathrm{T}_{\mathrm{a}}$ of $32^{\circ} \mathrm{C}$. (a) RTPCR analysis of AQP expression in the submandibular glands of rats kept at a $\mathrm{T}_{\mathrm{a}}$ of $24^{\circ} \mathrm{C}$ or $32^{\circ} \mathrm{C}$ for 5 days. (b) Relative levels of AQP1 mRNA in the submandibular glands of rats kept at a $\mathrm{T}_{\mathrm{a}}$ of $24^{\circ} \mathrm{C}$ or $32^{\circ} \mathrm{C}$ for 5 days. Values are the means $\pm \mathrm{SEM} . * \mathrm{P}<0.05$. (c) Relative levels of AQP5 mRNA in the submandibular glands of rats kept at a $\mathrm{T}_{\mathrm{a}}$ of $24^{\circ} \mathrm{C}$ or $32^{\circ} \mathrm{C}$ for 5 days. Values are the means $\pm \mathrm{SEM}$. $* * \mathrm{P}<0.01$. 
at 5 days after continuous heat exposure compared with that in the control rats (Fig. 4A, B). Similarly to AQP1 immunoreactivity, CD31-positive capillaries increased in the submandibular glands at 5 days after continuous heat exposure compared with that in the control rats (Fig. 5A, B), indicating that continuous heat exposure might promote an increase of capillaries in the submandibular glands.

However, the details regarding the mechanisms and role underlying heat regulation of AQP1 and angiogenesis remain unknown. Further studies are required to determine the mechanism and role of heat-dependent regulation of AQP1 expression in the submandibular glands of rats. Thus, we investigated the role of the transcription factor HIF-1 on heat acclimation because HIF-1 has been described to induce angiogenesis ${ }^{19}$.

Expression of HIF-1 $\alpha$ and VEGF in submandibular glands. To test whether HIF- $1 \alpha$ and VEGF are involved in the continuous heat exposure-induced changes, we examined the expression of HIF- $1 \alpha$ and VEGF mRNAs in submandibular glands by RT-PCR. HIF- $1 \alpha$ and VEGF expression were detectable in the control and heatexposed rats, and the mRNA levels of the HIF-1 $\alpha$ and VEGF increased at 5 days after continuous heat exposure in rats (Fig. 6A). To confirm the results of RT-PCR, we next examined the expression of VEGF in submandibular glands by western blotting. As expected, the VEGF protein level increased at 5 days after continuous heat exposure (Fig. 6B). These results suggest that continuous heat exposure may regulate the expression of HIF- $1 \alpha$ and
VEGF to induce angiogenesis in the submandibular glands of rats (Fig. 5A, B).

Immunohistochemistry detected VEGF protein in the cytosol and apical membranes of the duct cells in rat submandibular glands (Fig. 6C). Consistent with the expression of VEGF protein in western blot analyses (Fig. 6B), VEGF immunoreactivity in the cytosol and apical membranes of the duct cells increased and became more evident at 5 days after continuous heat exposure compared with that in the control rats (Fig. 6C). These results suggest that continuous heat exposure might induce VEGF expression via the induction of HIF- $1 \alpha$.

Cellular distribution of AQP5 in the submandibular glands of rats. Immunohistochemistry using an AQP5-specific antibody detected AQP5 protein expression in the submandibular glands of rats, including serous and mucous acinar cells (Fig. 7A). AQP5 was considerably localized at the apical membranes in the serous acini of submandibular glands and was also detected at the basolateral membranes of the mucous acini (Fig. 7A). Interestingly, AQP5 immunoreactivity at the apical membranes of the serous acini increased and became more evident at 5 days after continuous heat exposure compared with that in the control rats (Fig. 7A, B). AQP5 immunoreactivity at the basolateral membranes of the mucous acini did not differ between the continuously heat-exposed rats and control rats (Fig. 7A). The duct cells did not show AQP5 immunoreactivity in the rat submandibular glands. These results suggest that continuous heat exposure induces strong expression of

\section{a Western blot}

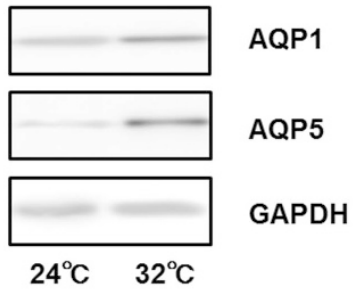

b

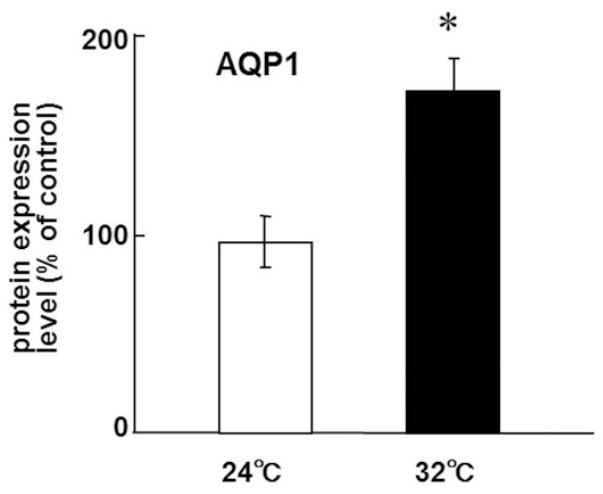

C

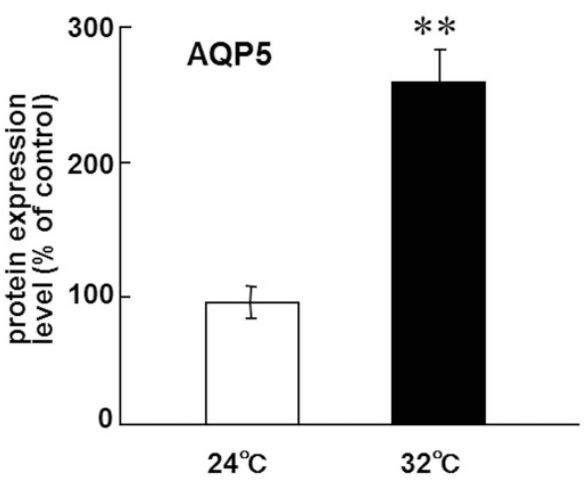

Figure $3 \mid$ Protein expression of AQP1 and AQP5 in rat submandibular glands are upregulated after 5 days of continuous exposure to a $\mathrm{T}_{\mathrm{a}}$ of $32^{\circ} \mathrm{C}$. (a) Western blotting of AQP1 and AQP5 in the submandibular glands of rats kept at a $\mathrm{T}_{\mathrm{a}}$ of $24^{\circ} \mathrm{C}$ or $32^{\circ} \mathrm{C}$ for 5 days. (b) Relative levels of protein in the submandibular glands of rats kept at a $\mathrm{T}_{\mathrm{a}}$ of $24^{\circ} \mathrm{C}$ or $32^{\circ} \mathrm{C}$ for 5 days. Values are the means \pm SEM. $* \mathrm{P}<0.05$. (c) Relative levels of AQP5 protein in the submandibular glands of rats kept at a $\mathrm{T}_{\mathrm{a}}$ of $24^{\circ} \mathrm{C}$ or $32^{\circ} \mathrm{C}$ for 5 days. Values are the means $\pm \mathrm{SEM}$. $* * \mathrm{P}<0.01$. 


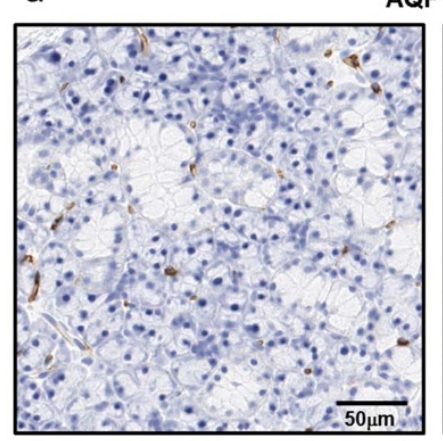

b

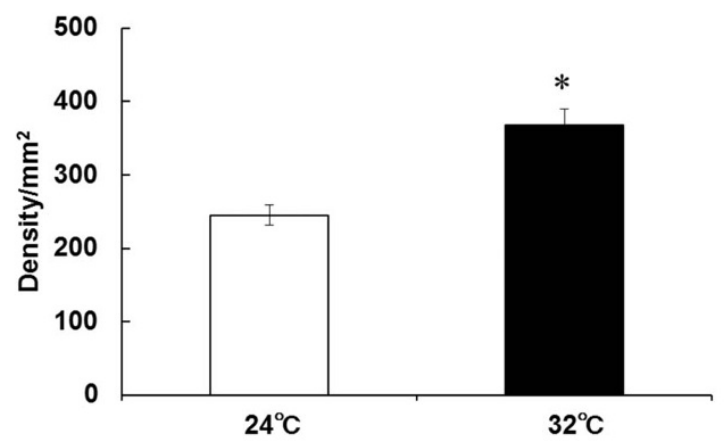

Figure $4 \mid$ AQP1-positive cells in rat submandibular glands are increased after 5 days of continuous exposure to a $\mathrm{T}_{\mathrm{a}}$ of $32^{\circ} \mathrm{C}$. (a) Representative images of AQP1 staining of submandibular gland sections from rats kept at a $\mathrm{T}_{\mathrm{a}}$ of $24^{\circ} \mathrm{C}$ or $32^{\circ} \mathrm{C}$ for 5 days. (b) Density of AQP1-positive cells in submandibular gland sections from rats kept at a $\mathrm{T}_{\mathrm{a}}$ of $24^{\circ} \mathrm{C}$ or $32^{\circ} \mathrm{C}$ for 5 days. Values are the means \pm SEM. $* \mathrm{P}<0.05$.

AQP5 at the apical membranes in the serous acini of submandibular glands.

\section{Discussion}

The main findings of this study are that 5 days of continuous heat exposure promoted the induction of HSP60 and HSP70, upregulated the expression of AQP1 and AQP5, and enhanced the apical membrane distribution of AQP5 in the serous acinar cells of the rat submandibular gland. In addition, activation of HIF- $1 \alpha$ by heat induced the expression of VEGF and promoted the distribution of CD31positive endothelial cells in the submandibular glands of rats. These results suggest that the role of heat in the molecular secretory mechanism might be associated with regulation of AQP expression, activation of HIF- $1 \alpha$-VEGF signaling, and promotion of angiogenesis in the submandibular glands.

Heat acclimation is an adaptive physiological process that occurs in humans and animals by repeated exposure to hyperthermia such as that at a high $\mathrm{T}_{\mathrm{a}}{ }^{32,33}$. The primary benefit of heat acclimation is improved heat tolerance. It is accepted that sufficient adaptation to heat stress occurs after 4 to 6 days $s^{31,34,35}$. Indeed, 5 days of continuous exposure to heat stress significantly attenuates thermal strain and enhances exercise capacity in the heat, and these findings concur with those of previous reports in humans and animals ${ }^{31,34,35}$. Our results indicate that 5 days of continuous exposure to heat stress regulates HSP levels in rats, as well as heat acclimation of animals as reported previously ${ }^{6}$. Interestingly, we have recently reported that 5 days of continuous exposure to mild heat stress attenuates heat shock-induced apoptosis and improves heat tolerance in cultured cells, concomitant with the upregulation of $\mathrm{HSPs}^{36}$. Taken together, a

CD31
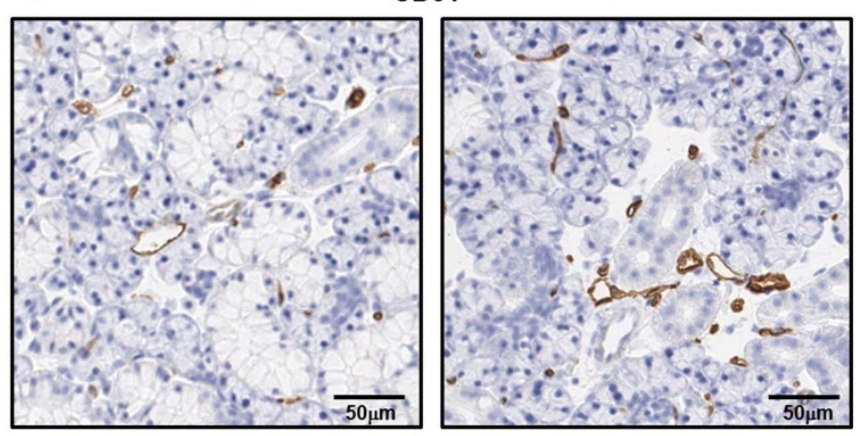

b

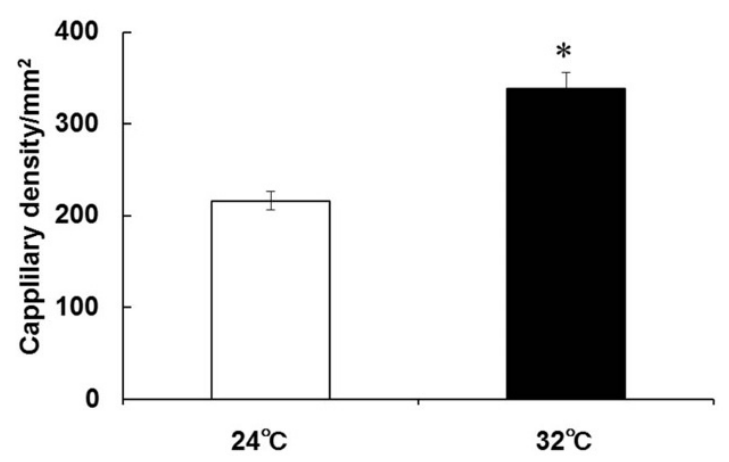

Figure 5 Capillaries in rat submandibular glands are increased after 5 days of continuous exposure to a $\mathrm{T}_{\mathrm{a}}$ of $32^{\circ} \mathrm{C}$. (a) Representative images of capillaries stained with an anti-CD31 antibody in submandibular glands section from rats kept at a $\mathrm{T}_{\mathrm{a}}$ of $24^{\circ} \mathrm{C}$ or $32^{\circ} \mathrm{C}$ for 5 days. (b) Density of capillaries in submandibular glands sections from rats kept at a $\mathrm{T}_{\mathrm{a}}$ of $24^{\circ} \mathrm{C}$ or $32^{\circ} \mathrm{C}$ for 5 days. Values are the means \pm SEM. ${ }^{*} \mathrm{P}<0.05$. a

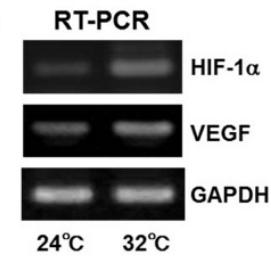

C

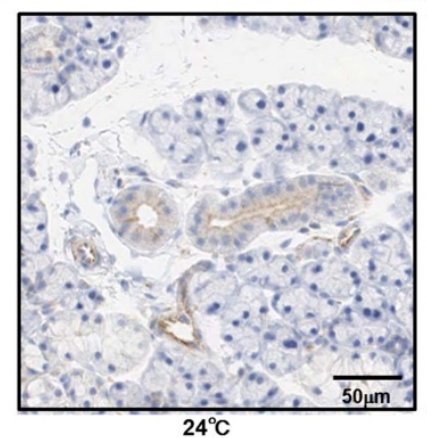

b western blot

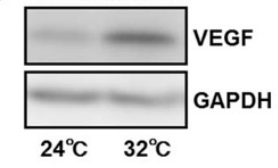

VEGF

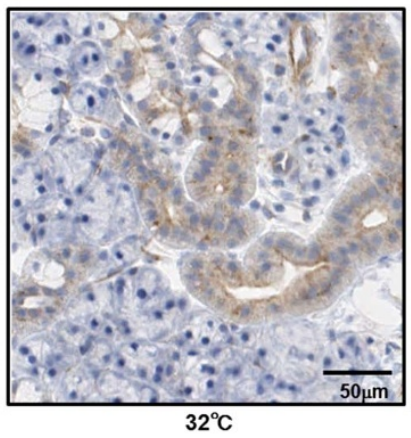

Figure 6 | HIF-1 and VEGF expression in rat submandibular glands are upregulated after 5 days of continuous exposure to a $T_{a}$ of $32^{\circ} \mathrm{C}$. (a) RTPCR analysis of HIF-1 and VEGF expression in the submandibular glands of rats kept at a $\mathrm{T}_{\mathrm{a}}$ of $24^{\circ} \mathrm{C}$ or $32^{\circ} \mathrm{C}$ for 5 days. (b) Western blotting of VEGF in the submandibular glands of rats kept at a $\mathrm{T}_{\mathrm{a}}$ of $24^{\circ} \mathrm{C}$ or $32^{\circ} \mathrm{C}$ for 5 days. (c) Representative images of duct cells stained with an anti-VEGF antibody in submandibular gland sections from rats kept at a $\mathrm{T}_{\mathrm{a}}$ of $24^{\circ} \mathrm{C}$ or $32^{\circ} \mathrm{C}$ for 5 days. 
a

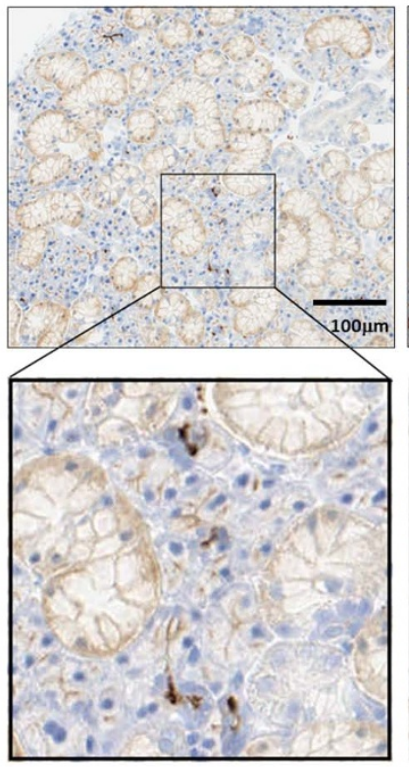

$24^{\circ} \mathrm{C}$

\section{AQP5}

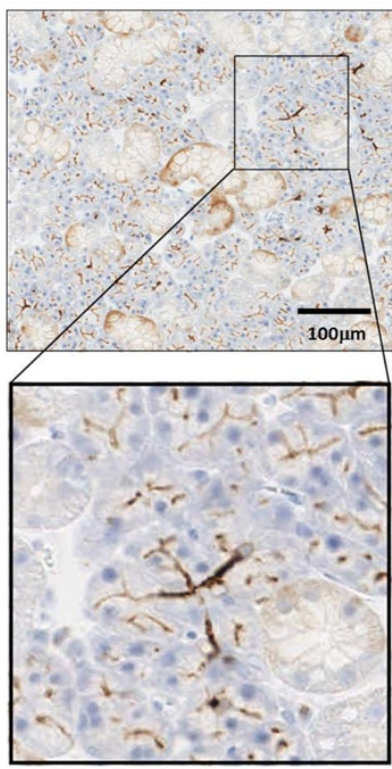

$32^{\circ} \mathrm{C}$ b

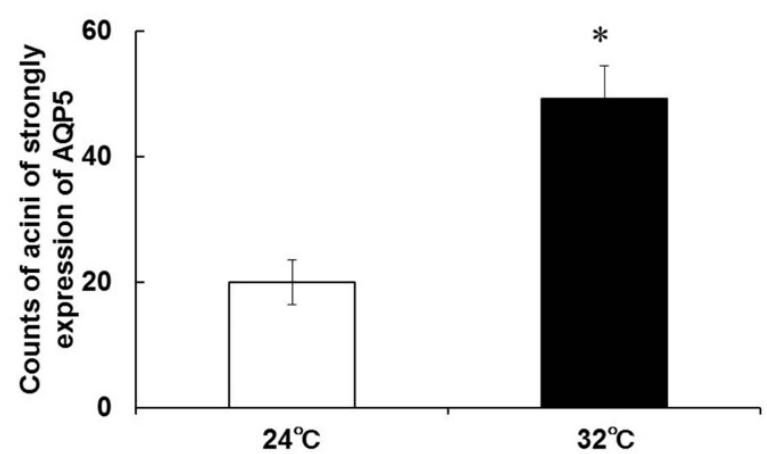

Figure $7 \mid$ AQP5-positive cells in rat submandibular glands are increased after 5 day continuous exposure to $\mathrm{T}_{\mathrm{a}}$ of $32^{\circ} \mathrm{C}$. (a) Representative images of AQP5 staining in submandibular gland sections from rats kept at a $\mathrm{T}_{\mathrm{a}}$ of $24^{\circ} \mathrm{C}$ or $32^{\circ} \mathrm{C}$ for 5 days. (b) Counts of acini in strongly AQP5-positive cells in submandibular glands sections from rats kept at a $\mathrm{T}_{\mathrm{a}}$ of $24^{\circ} \mathrm{C}$ or $32^{\circ} \mathrm{C}$ for 5 days. Values are the means \pm SEM. ${ }^{*} \mathrm{P}<0.05$.

our results suggest that 5 days of continuous exposure to heat stress may induce molecular adaptations to tolerate the heat, including HSPs, in animals as well as in cultured cells.

AQP5 is highly expressed in the apical membranes of salivary acinar cells, and it plays a major role in rapid movement of water in salivary glands ${ }^{14,15}$. Thus, downregulation of AQP5 expression in salivary glands results in salivary gland hyposecretion ${ }^{9,10}$. Based on these evidences, upregulation of AQP5 expression in salivary glands may lead to hypersecretion. The mRNA and protein expression of AQP5 in the submandibular glands was shown to increase after 2 days (data not shown) and 5 days (Figs. 2, 3) of heat exposure in our study. Saliva secretion is known to be affected by neurohumoral (extracellular) factors as well as the water permeability of acinar cells. Kaspler and Horowitz have shown that extracellular factors might regulate the originating cellular responses elicited by the persistent heat stress in heat-acclimated animals ${ }^{37}$. Thus, the saliva secretion with upregulation of water permeability of acinar cells might be affected by extracellular factors in the heat-acclimated rats. Further studies are required to determine the relationships between cellular water permeability and extracellular factors in heat-acclimated animals.

While the molecular link connecting heat acclimation to altered AQP5 expression remains unclear, one important element may be the HIF pathway. HIF-1 is a transcription factor that mediates adaptive responses to changes in tissue oxygenation. It regulates the transcription of numerous genes involved in vascular development, glucose and energy metabolism, as well as cell proliferation and viability. HIF-1 is a basic helix-loop-helix transcription factor composed of two subunits, HIF- $1 \alpha$ and HIF- $1 \beta^{20}$. HIF- $1 \beta$ is constitutively expressed, while the level of HIF- $1 \alpha$ is regulated by hypoxia and iron chelators. Previous reports have shown that HIF-1 $\alpha$ induction is necessary for hypertonic induction of AQP5 expression in rats $^{38}$. Two putative cis-acting HREs have been identified in the proximal rat AQP5 promoter/enhancer region, suggesting the possibility that the effects of hypertonic stress are mediated by the interaction of HIF-1 with the proximal rat AQP5 promoter. In this study, we found heat acclimation induced the expression of HIF-1 $\alpha$ (Fig. 6) concomitantly with the induction of AQP5 (Figs. 1, 2, and 7) in rat submandibular glands. Interestingly, in Caenorhabditis elegans, recent studies have demonstrated that HIF- $1 \alpha$ knockout negates acclimation to heat, suggesting that HIF- $1 \alpha$ is essential for heat acclimation $^{39}$. Additionally, in mammalian species, constitutive upregulation of HIF- $1 \alpha$ occurs in the hearts and brains of heat-acclimated animals ${ }^{40,41}$, consistent with our present observation that continuous heat exposure elevated the level of HIF- $1 \alpha$ mRNA in rat submandibular glands (Fig. 6). In an additional experiment (Fig. 8), we showed that $5 \% \mathrm{O}_{2}$ hypoxia induced upregulation of AQP5 mRNA in cultured mouse fibroblasts in which continuous mild heat stress has recently been shown to induce expression of $\mathrm{AQP} 5^{36}$. Therefore, we speculate that heat-induced expression of AQP5 mRNA in the submandibular gland might involve the HIF-1 pathway.

\section{RT-PCR}
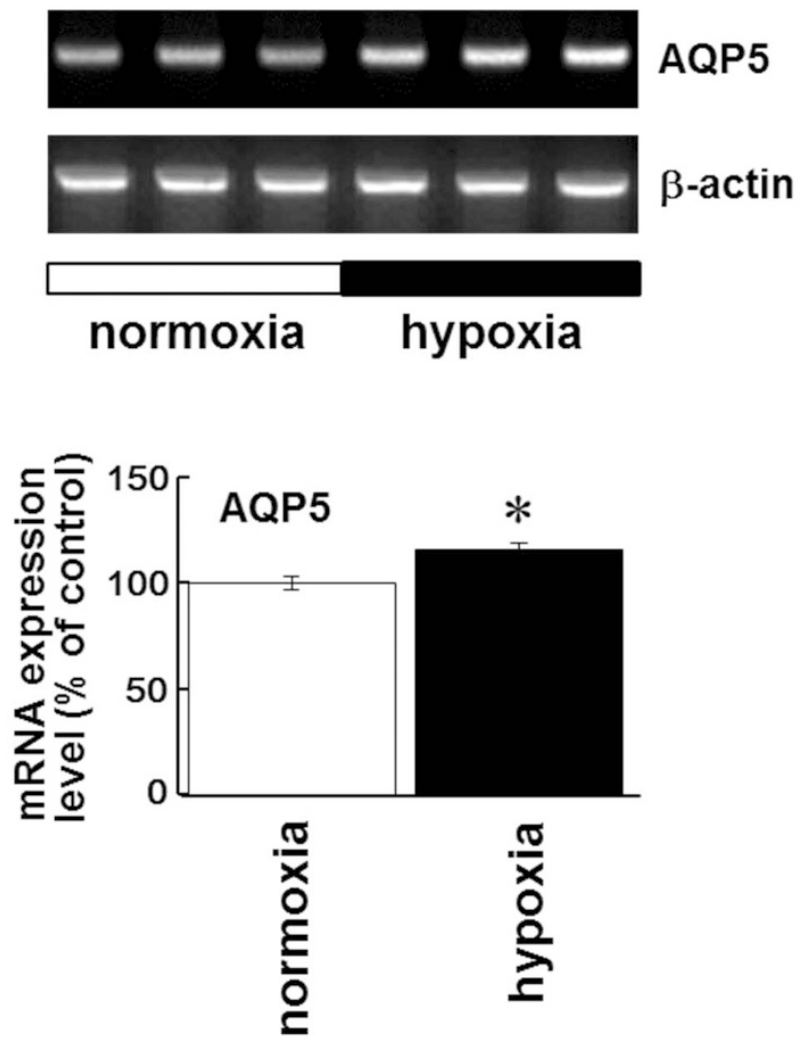

Figure 8 Five percent $\mathrm{O}_{2}$ hypoxia induces AQP5 expression in mouse fibroblasts. (a) RT-PCR analysis of AQP5 expression in mouse fibroblasts kept in a $20 \% \mathrm{O}_{2}$ (normoxia) or $5 \% \mathrm{O}_{2}$ (hypoxia) incubator for 5 days. (b) Relative levels of AQP5 mRNA in mouse fibroblasts kept in a $20 \% \mathrm{O}_{2}$ (normoxia) or $5 \% \mathrm{O}_{2}$ (hypoxia) incubator for 5 days. Values are the means \pm SEM. $* \mathrm{P}<0.05$. 
In addition to the direct effects of heat on the regulation of AQP5 and HIF- $1 \alpha$ induction, we also inferred the effects of heat on blood flow in the submandibular glands, because blood flow is an important factor influencing the secretion of exocrine glands. Any reduction of blood flow might reduce secretory responses ${ }^{42,43}$. In contrast, an improvement in the blood flow in the submandibular glands can increase the salivary secretion ${ }^{44}$. Angiogenesis, as well as local vasodilation, usually results in an increase of blood flow in tissue ${ }^{45}$. As discussed above, heat acclimation induces HIF- $1 \alpha$, which is linked to VEGF expression, in the heart and brain ${ }^{40,41}$ as well as the submandibular glands (Fig. 6). VEGF has been demonstrated to be a major contributor to angiogenesis by increasing the number of capillaries in a given network ${ }^{20}$. We found increased VEGF induction (Fig. 6) and CD31-positive capillaries (Fig. 5) in the submandibular glands after heat acclimation. In rat salivary glands, $\mathrm{AQP} 1$ expression is restricted to the endothelial cells of the microvasculature, suggesting that AQP1 probably contributes to the water permeability of the microvasculature from plasma across the endothelial barrier ${ }^{46,47}$. In this study, continuous heat exposure increased the number of AQP1positive cells that may be endothelial cells at the capillaries (Fig. 4A, B). These results suggest that heat-induced salivation is due to an enhancement of blood flow in heat-acclimated rats.

Lastly, AQP8 is reportedly expressed in the salivary glands, although there are discrepancies among the data. Koyama et al. showed the expression and localization of AQP8 mRNA in the acinar cells of rat salivary glands by in situ hybridization ${ }^{48}$, whereas Ishibashi et al. failed to detect AQP8 mRNA in the rat salivary gland by northern blot analysis ${ }^{49}$. In this study, we detected AQP8 mRNA in rat submandibular glands by RT-PCR analysis with specific primers for rat AQP8 mRNA. Furthermore, heat acclimation did not affect the expression of AQP8 mRNA, but did affect AQP5 and AQP1 mRNA levels, in the salivary glands. However, we did not investigate the expression and localization of AQP8 protein in rat submandibular glands, because an anti-AQP8 antibody was not available for the detection of rat AQP8 in this study. Therefore, further analysis will be required to determine the expression and localization of AQP8 in the rat submandibular glands.

Our present report revealed the part of the molecular mechanisms of regulation of salivary secretion after heat acclimation. The molecular mechanism of salivation will provide further understanding of the physiological salivary function and useful insights into novel anti-hyposecretion therapy.

\section{Methods}

All animal experiments were performed in accordance with the Guidelines for Animal Experimentation of Shimane University Faculty of Medicine, which were compiled from the Guidelines for Animal Experimentation of the Japanese Association for Laboratory Animal Science.

Animals. Male Wistar rats (5 weeks of age) were housed individually in transparent plastic cages (width, $270 \mathrm{~mm}$; length, $440 \mathrm{~mm}$; height, $187 \mathrm{~mm}$ ) with wood chippings, and were initially maintained at a $\mathrm{T}_{\mathrm{a}}$ of $24.0 \pm 0.1^{\circ} \mathrm{C}$ with $54 \pm 5 \%$ relative humidity under a $12: 12$-h light-dark cycle (lights on at 1600 hours). Because the rats were kept in plastic cages, air velocity inside the cages was negligible. For heat acclimation, rats were subjected to a constant $\mathrm{T}_{\mathrm{a}}$ of $32.0 \pm 0.2^{\circ} \mathrm{C}$ with $35 \pm 8 \%$ relative humidity for 5 days, whereas control rats were continuously kept at $24.0 \pm 0.1^{\circ} \mathrm{C}$ with $54 \pm 5 \%$ relative humidity. The $\mathrm{T}_{\mathrm{a}}$ for control rats (and heat acclimation rats before heat exposure) was selected because freely moving Wistar rats have been reported to prefer about 24 to $26^{\circ} \mathrm{C}^{50}$. During the experiment, cages were cleaned, and food and water were replaced every 2 or 3 days at random times of the day.

Chemicals. Dulbecco's modified Eagle's medium (DMEM) was obtained from Wako Pure Chemical Industries, Ltd (Osaka, Japan). Fetal bovine serum (FBS) was obtained from Invitrogen Corporation (Carlsbad, CA). Rabbit anti-HSP60, rabbit anti-HSP70, rabbit anti-GAPDH, horseradish peroxidase (HRP)-linked goat anti-rabbit IgG, and horse anti-mouse IgG were purchased from Cell Signaling Technology, Inc. (Danvers, MA). Rabbit anti-AQP1 and rabbit anti-VEGF were obtained from Santa Cruz Biotechnology (Santa Cruz, CA). Rabbit anti-AQP5 was obtained from Calbiochem (La Jolla, CA). Mouse anti-CD31 was obtained from DAKO (Glostrup, Denmark). Biotinylated goat anti-rabbit IgG antibody, biotinylated horse anti-mouse IgG antibody, and streptavidin-conjugated peroxidase were purchased from Vector Laboratories (Burlingame, CA)

Western blot analysis. On days 2 and 5 of the heat exposure period, the animals were anesthetized with pentobarbital sodium ( $50 \mathrm{mg} / \mathrm{kg}$, i.p.) and perfused transcardially with PBS. Submandibular glands were removed and homogenated in RIPA buffer by a glass homogenizer. After removal of the tissue debris by centrifugation at $800 \mathrm{~g}$ for $15 \mathrm{~min}$ at $4^{\circ} \mathrm{C}$, the supernatants were analyzed by western blotting as described previously ${ }^{51}$. Briefly, proteins were extracted from the submandibular glands, and protein concentrations were determined by a protein assay. Equal amounts of protein were separated by $10 \%$ or $20 \%$ sodium dodecyl sulfate-polyacrylamide gel electrophoresis. The resolved proteins were transferred onto polyvinylidene fluoride membranes that were incubated with primary antibodies $(1: 1000)$ and then HRPlinked secondary antibodies $(1: 2000)$. The blots were developed using Immobilon Western Chemiluminescence HRP Substrate (Millipore, Billerica, MA).

Reverse transcription-polymerase chain reaction analysis. On days 5 of the heat exposure period, the animals were anesthetized with pentobarbital sodium (50 mg/ $\mathrm{kg}$, i.p.) and perfused transcardially with PBS. Submandibular glands were removed for RT-PCR. To evaluate the mRNA expression profiles of AQPs, HIF-1 $\alpha$, and VEGF in submandibular glands or cells, RT-PCR was performed as follows. Briefly, RNA was extracted from the submandibular glands or cells, and reverse transcribed by using a reverse transcription kit (RR037A; TAKARA, Tokyo, Japan). RT-PCR analysis of AQP1, AQP3, AQP4, AQP5, AQP6, AQP8, AQP9, GAPDH, HIF- $1 \alpha$, VEGF and $\beta$-actin mRNA levels was performed with LA Taq (TAKARA, Tokyo, Japan) by using the following primers: $5^{\prime}$-catgtacatcatcgcccagt- $3^{\prime}$ and $5^{\prime}$ ccacagccagtgtagtcaat- $3^{\prime}$ for AQP1, $5^{\prime}$-agatgctccacatccgctac- $3^{\prime}$ and $5^{\prime}$ agatgggcagcttgatccag- $3^{\prime}$ for AQP3, $5^{\prime}$-tgctttggactcagcattgc- $3^{\prime}$ and $5^{\prime}$ cgtttggaatcacagctggca- $3^{\prime}$ for AQP4, 5' -gcccagctggtgggcgccatt- $3^{\prime}$ and $5^{\prime}$ tggggagcccacagggctggt- $3^{\prime}$ for AQP5, 5' -gggatctacttcactggctg- $3^{\prime}$ and $5^{\prime}$ ggtcttggtgtcagggaaca- $3^{\prime}$ for AQP6, 5' -gtatggacctacgcgagatca- $3^{\prime}$ and $5^{\prime}$ ctgatgttccccaaggtagca- $3^{\prime}$ for AQP8, 5' -cacatcaacccagctgtgtc- $3^{\prime}$ and $5^{\prime}$ cccaggtttctggagtcaaa- $3^{\prime}$ for AQP9, $5^{\prime}$-tgcaccaccaactgcttagc- $3^{\prime}$ and $5^{\prime}$ ggatgcagggatgatgttctg- $3^{\prime}$ for GAPDH, $5^{\prime}$-gaactaactggacacagtgtg- $3^{\prime}$ and $5^{\prime}$ ggaatgggttcacaaatcagc- $3^{\prime}$ for HIF- $1 \alpha, 5^{\prime}$-cctggctttactgctgtacct- $3^{\prime}$ and $5^{\prime}$ gatgtccaccagggtctcaat- $3^{\prime}$ for VEGF, and $5^{\prime}$-atggtgggtatgggtcagaag- $3^{\prime}$ and $5^{\prime}$ ctggggtgttgaaggtctcaa- $3^{\prime}$ for $\beta$-actin.

Immunohistochemistry. On day 5 of the heat exposure period, the animals were anesthetized with pentobarbital sodium $(50 \mathrm{mg} / \mathrm{kg}$, i.p.) and perfused transcardially with PBS. Submandibular glands were removed, fixed overnight at $4{ }^{\circ} \mathrm{C}$ in $4 \%$ formaldehyde, and then immersed in a $20 \%(\mathrm{w} / \mathrm{v})$ sucrose solution. The submandibular gland samples were then analyzed by immunohistochemistry as described previously ${ }^{52}$. Formalin-fixed paraffin-embedded sections of submandibular glands were deparaffinized and subjected to a $10 \mathrm{mM}$ sodium citrate buffer, $\mathrm{pH}$ 6.0, for antigen retrieval. Sections were then incubated in DAKO Protein Block (DAKO, Mississauga, ON, Canada) for 1 hour. Antigen detection was performed sequentially by primary antibodies $(1: 100)$, a biotinylated anti-IgG antibody, and streptavidinconjugated peroxidase, and visualized with $3,3^{\prime}$-diaminobenzidine

tetrahydrochloride. AQP1 and capillary densities were counted in randomly chosen high-power fields per mouse and expressed as the number of capillaries per $\mathrm{mm}^{2}$. Acini of strongly expression of AQP5 were counted in randomly chosen high-power fields per mouse and expressed as the number of capillaries per $0.25 \mathrm{~mm}^{2}$.

Cell culture and hypoxic condition. NIH3T3 mouse fibroblasts were provided by Dr. Komine (Kanazawa University). The cells were maintained in DMEM containing $10 \% \mathrm{FBS}$ at $37^{\circ} \mathrm{C}$ in a $5 \% \mathrm{CO}_{2}$ and $20 \% \mathrm{O}_{2}$ incubator. Then, the cells were divided into 2 groups. The cells for normoxia were kept in a $5 \% \mathrm{CO}_{2}$ and $20 \% \mathrm{O}_{2}$ incubator, while the cells for hypoxia were maintained in a $5 \% \mathrm{CO}_{2}$ and $5 \% \mathrm{O}_{2}$ incubator for 5 days.

Statistical analysis. Statistical analyses were performed using Student's unpaired ttest. All the data are expressed as mean \pm SEM.

1. McCutcheon, L. J. \& Geor, R. J. Influence of training on sweating responses during submaximal exercise in horses. J Appl Physiol 89, 2463-2471 (2000).

2. Samuel, N. \& Cheuvront, S. N. et al. Mechanisms of aerobic performance impairment with heat stress and dehydration. J Appl Physiol 109, 1989-1995 (2010).

3. Gisolfi, C. V. et al. Effects of changing hypothalamic temperature on eccrine sweating in the patas monkey. Brain Res Bul 20, 179-182 (1988).

4. Robertshaw, D. Mechanisms for the control of respiratory evaporative heat loss in panting animals. J Appl Physiol 101, 664-668 (2006).

5. Sugimoto, N. et al. Thermoregulatory responses to acute heat loads in the FOK rat. Int J Biometeorol 43, 199-123 (1999).

6. Furuyama, F. et al. Differences in thermal salivation between the FOK rat (a model of genotypic heat adaptation) and three other rat strains. Physiol Behav 63, 787-793 (1998)

7. Horowitz, M. \& Meiri, U. Altered responsiveness to parasympathetic activation of submaxillary salivary gland in the heat-acclimated rat. Comp Biochem Physiol A Comp Physiol 80, 57-60 (1985). 
8. Whyte, D. G., Brennan, T. J. \& Johnson, A. K. Thermoregulatory behavior is disrupted in rats with lesions of the anteroventral third ventricular area (AV3V). Physiol Behav 87, 493-499 (2006).

9. Krane, C. M. et al. Salivary acinar cells from aquaporin 5-deficient mice have decreased membrane water permeability and altered cell volume regulation. J Biol Chem 276, 23413-23420 (2001).

10. Ma, T. et al. Defective secretion of saliva in transgenic mice lacking aquaporin-5 water channels. J Biol Chem 274, 20071-20074 (1999).

11. Farnaud, S. J. et al. Saliva: physiology and diagnostic potential in health and disease. Scientific World Journal 10, 434-456 (2010).

12. Humphrey, S. P. \& Williamson, R. T. A review of saliva: Normal composition, flow, and function. J Prosth Dent 85, 162-169 (2001).

13. Matsuzaki, T. et al. Aquaporins in the digestive system. Med Electron Microsc 37, 71-80 (2004)

14. Borgnia, M. et al. Cellular and molecular biology of the aquaporin water channels. Annu Rev Biochem 68, 425-458 (1999).

15. Verkman, A. S. \& Mitra, A. K. Structure and function of aquaporin water channels. Am J Physiol Renal Physiol 278, F13-F28 (2000).

16. Agre, P. et al. Aquaporin water channels--from atomic structure to clinical medicine. J Physiol 541, 3-16 (2002).

17. King, L. S., Kozono, D. \& Agre, P. From structure to disease: the evolving tale of aquaporin biology. Nat Rev Mol Cell Biol 5, 687-698 (2004).

18. Ishibashi, K. New members of mammalian aquaporins: AQP10-AQP12. Handb Exp Pharmacol 190, 251-262 (2009).

19. Richard, D. E., Berra, E. \& Pouysségur, J. Angiogenesis: how a tumor adapts to hypoxia. Biochem Biophys Res Commun 266, 718-722 (1999).

20. Semenza, G. L. Hypoxia-inducible factors: mediators of cancer progression and targets for cancer therapy. Trends Pharmacol Sci 33, 207-214 (2012).

21. Akamatsu, T. et al. Expression and localization of aquaporins, members of the water channel family, during development of the rat submandibular gland. Pflügers Arch 446, 641-651 (2003).

22. Gresz, V. et al. Identification and localization of aquaporin water channels in human salivary glands. Am J Physiol Gastrointest Liver Physiol 281, G247-G254 (2001).

23. Steinfeld, S. et al. Abnormal distribution of aquaporin-5 water channel protein in salivary glands from Sjögren's syndrome patients. Lab Invest 81, 143-148 (2001).

24. Horowitz, M. \& Assadi, H. Heat acclimation-mediated cross-tolerance in cardioprotection. Do HSP70 and HIF- $1 \alpha$ play a role? Ann NY Acad Sci 1188, 199-206 (2010).

25. Morimoto, R. I. \& Santoro, M. G. Stress-inducible responses and heat shock proteins: new pharmacologic targets for cytoprotection. Nat Biotech 16, 833-838 (1998).

26. Creagh, E. M., Sheehan, D. \& Cotter, T. G. Heat shock proteins--modulators of apoptosis in tumour cells. Leukemia 14, 1161-1173 (2000).

27. Maloyan, A., Palmon, A. \& Horowitz, M. Heat acclimation increases the basal HSP72 level and alters its productin dynamics during heat stress. Am J Physiol 276, R1506-R1515 (1999).

28. Horowitz, M. \& Meiri, U. Altered responsiveness to parasympathetic activation of submaxillary salivary gland in the heat-acclimated rat. Comp Biochem Physiol A Comp Physiol 80, 5-60 (1985).

29. Horowitz, M., Argov, D. \& Mizrahi, R. Interrelationships between heat acclimation and salivary cooling mechanism in conscious rats. Comp Biochem Physiol A Comp Physiol 74, 945-949 (1983).

30. Horowitz, M. \& Soskolne, W. A. Cellular dynamics of rats' submaxillary gland during heat acclimatization. J Appl Physiol 44, 21-24 (1978).

31. Sareh, H. et al. Response of mice to continuous 5-day passive hyperthermia resembles human heat acclimation. Cell Stress Chaperones 16, 297-307 (2011)

32. McClung, J. P. et al. Exercise-heat acclimation in humans alters baseline levels and ex vivo heat inducibility of HSP72 and HSP90 in peripheral blood mononuclear cells. Am J Physiol Regul Integr Comp Physiol 294, R185-R191 (2008).

33. Sawka, M. N. et al. Altitude acclimatization and blood volume: effects of exogenous erythrocyte volume expansion. J Appl Physiol 81, 636-642 (1996).

34. Garrett, A. T. et al. Induction and decay of short-term heat acclimation. Eur J Appl Physiol 107, 659-670 (2009).

35. Horowitz, M. \& Konesh, E. Molecular signals that shape the integrative responses of the heat-acclimated phenotype. Med Sci Sports Exerc 42, 2164-2172 (2010).

36. Sugmoto, N. et al. Cellular Heat Acclimation Regulates Cell Growth, Cell Morphology, Mitogen-activated Protein Kinase Activation, and Expression of Aquaporins in Mouse Fibroblast Cells. Cell Physiol Biochem 30, 450-457 (2012).
37. Kaspler, P. \& Horowitz, M. Heat acclimation and heat stress have different effects on cholinergic muscarinic receptors. Ann N Y Acad Sci 813, 620-627 (1997)

38. Zhou, B. et al. Hypertonic induction of aquaporin-5: novel role of hypoxiainducible factor-1alpha. Am J Physiol Cell Physiol 292, C1280-1290 (2007).

39. Treinin, M. et al. HIF-1 is required for heat acclimation in the nematode Caenorhabditis elegans. Physiol Genomics 14, 17-24 (2003).

40. Maloyan, A. et al. HIF-1alpha-targeted pathways are activated by heat acclimation and contribute to acclimation-ischemic cross-tolerance in the heart. Physiol Genomics 23, 79-88 (2005).

41. Shein, N. A. et al. Heat acclimation increases hypoxia-inducible factor 1alpha and erythropoietin receptor expression: implication for neuroprotection after closed head injury in mice. J Cereb Blood Flow Metab 25, 1456-1465 (2005).

42. Garrett, J. R. et al. The proper role of nerves in salivary secretion: a review. J Dent Res 66, 387-397 (1987).

43. Thakor, A. S., Brown, C. N. \& Edwards, A. V. Effects of prolonged reduction in blood flow on submandibular secretory function in anesthetized sheep. J Appl Physiol 95, 751-757 (2003)

44. Yu, G. Y. et al. Microvascular autologous submandibular gland transfer in severe cases of keratoconjunctivitis sicca. Microvascular autologous submandibular gland transfer in severe cases of keratoconjunctivitis sicca. Int J Oral Maxillofac Surg 33, 235-239 (2004).

45. Oyama, O. et al. The lysophospholipid mediator sphingosine-1-phosphate promotes angiogenesis in vivo in ischaemic hindlimbs of mice. Cardiovasc Res 78, 301-307 (2008)

46. Li, J. et al. Examination of rat salivary glands for the presence of the aquaporin CHIP. Pflügers Arch 428, 455-460 (1994).

47. Nielsen, S. et al. Distribution of the aquaporin CHIP in secretory and resorptive epithelia and capillary endothelia. Proc Natl Acad Sci USA 90, 7275-7279 (1993)

48. Koyama, Y. et al. Molecular cloning of a new aquaporin from rat pancreas and liver. J Biol Chem 272, 30329-30333 (1997).

49. Ishibashi, K. et al. Cloning and functional expression of a second new aquaporin abundantly expressed in testis. Biochem Biophys Res Commun 237, 714-718 (1997).

50. Sugimoto, N., Sakurada, S. \& Shido, O. Selected ambient temperatures of rats acclimated to heat given on various schedules. Pflügers Arch 438, 766-770 (1999)

51. Saito, T. et al. Phosphodiesterase inhibitors suppress Lactobacillus casei cell wallinduced NF- $\kappa \mathrm{B}$ and MAPK activations and cell proliferation through protein kinase A- or exchange protein activated by cAMP-dependent signal pathway. Scientific World Journal 2012, 748572 (2012).

52. Matsuzaki, K. et al. Proliferation of neuronal progenitor cells and neuronal differentiation in the hypothalamus are enhanced in heat-acclimated rats. Pflügers Arch 458, 661-673 (2009)

\section{Acknowledgment}

This work was supported in part by Grants-in-Aid for Science and Culture from the Ministry of Education, Culture, Sports, Science, and Technology of Japan.

\section{Author contributions}

N.S., O.S. and H.U. conceived the project. O.S., H.U., T.O., G.S., S.K., A.Y. and N.S. supervised all research. N.S., O.S. and H.U. wrote the manuscript. N.S., O.S. and K.M. designed the experiments. K.M., M.T., T.S., Y.F., T.K. and Y.M. performed the data analysis and produced the figures. K.M., N.S. and H.I. performed the experiments.

\section{Additional information}

Supplementary information accompanies this paper at http://www.nature.com/ scientificreports

Competing financial interests: The authors declare no competing financial interests.

License: This work is licensed under a Creative Commons

Attribution-NonCommercial-NoDerivs 3.0 Unported License. To view a copy of this license, visit http://creativecommons.org/licenses/by-nc-nd/3.0/

How to cite this article: Sugimoto, N. et al. Upregulation of aquaporin expression in the salivary glands of heat-acclimated rats. Sci. Rep. 3, 1763; DOI:10.1038/srep01763 (2013). 